\title{
Transient Analysis of Communication Network Model with Homogeneous Poisson arrivals and Dynamic Bandwidth Allocation
}

\author{
CH.V. Raghavendran \\ Research Scholar \\ Adikavi Nannayya \\ University, India
}

\author{
G. Naga Satish \\ Research Scholar \\ Adikavi Nannayya \\ University, India
}

\author{
M.V. Rama Sundari \\ Dept. of IT \\ GIET \\ Rajahmundry, India
}

\author{
P. Suresh Varma \\ Dept. of CS \\ Adikavi Nannayya \\ University, India
}

\begin{abstract}
Queuing models play a dominant role in many communication systems for optimum utilization of the resources. In this paper, we develop and analyze a two node tandem communication network model with feedback for the first node, with an assumption that the arrivals follow homogeneous Poisson process. In this model, the service rates of each transmitter depends on the number of services in the buffer connected it. The model is analyzed using the difference-differential equations and a probability generating function of the number of packets in the buffer. Expressions are derived for performance measures including average number of packets in each buffer, the probability of emptiness of the network, the mean delay in the buffer and in the network, the throughput of the transmitters, and the variance of the number of packets in the buffer.
\end{abstract}

\section{Keywords}

Tandem Communication Network, Dynamic Bandwidth Allocation, Performance Evaluation, Homogeneous Poisson process

\section{INTRODUCTION}

Queuing models are widely used in capacity planning and performance evaluation of computer and communication systems, service centers, manufacturing systems, etc. The low computational cost and adequate accuracy of queuing models in predicting the performance of computer systems has been generally established [1] [2] [3]. This is mainly due to their ability to model multiple independent resources and the sequential use of these resources by different jobs. Starting from the first queuing model by A.K. Erlang lot of work has been reported in literature regarding queuing models and their applications. In practical situations the output from one queuing process serves as input to other i.e., the queues are connected in series. These types of queuing systems are called tandem queuing systems.

Barden and Barttain have brought a tremendous revolution in communication network model. Later USA Network Group at MIT, IEEE communication society and other organizations has brought remarkable changes in designing efficient communication systems. For efficient design and evaluation of communication networks, the network models are developed and analyzed with various assumptions on constituent processes of the model like arrival process, service process, flow control mechanisms, allocations, routing, etc,.

Due to the several technological innovations in the recent years, a wide variety of communication networks are designed and analyzed with effective switching techniques. Based on the type of architecture, communication networks are divided into three categories - message switching, circuit switching and packet switching. To improve the Quality of Service, packet switching gives better utilization over circuit and message switching According to [4] [5], networks that support tele processing applications are mixed with dynamic engineering skills and statistical multiplexing. To improve the quality of service in transmission, several authors have studied the communication networks utilizing tandem queuing analogy [6] [7] [8].

In the literature, we found some observations regarding communication networks with dynamic bandwidth allocation/ load dependent transmission for improving quality of service by utilizing ideal bandwidth [9] [10] [11]. They considered that the arrivals of messages for transmission are homogeneous. In [9] a two node communication network with load dependent transmission is studied in which arrivals to the source node are single packets. The performance evaluation of a two node communication network with dynamic bandwidth allocation and modified phase type transmission having bulk arrivals is studied in [10]. In [12] a two node communication network with dynamic bandwidth allocation (DBA) having two stage bulk arrivals (BA) is introduced and analyzed. So, tandem queuing systems have found much interest in literature.

In many of the Satellite and Tele communication systems, the packet getting transmitted after the first transmitter returned back or forwarded to the second buffer connected to the second transmitter with certain probabilities. Conducting experiments with varying load conditions of a communication system in particular with DBA is difficult and complicated. So, mathematical models of communication networks are developed to evaluate the performance of the newly proposed communication network models under transient conditions.

The remainder of the paper is organized as follows. The section 2 describes the two node tandem communication network model the DBA and homogeneous passion arrivals with feedback for the first node. In section 3, the performance measures of the network model are derived and analyzed. In section 4 , the network model is evaluated with numerical illustration. The sensitivity analysis is described in the section 5. Section 6 consists of conclusion of the model along with its future scope.

\section{TANDEM COMMUNICATION NETWORK MODEL WITH DBA AND HOMOGENEOUS POISSON ARRIVALS WITH FEED BACK AT FIRST NODE}

Consider a communication network with two nodes connected in tandem. Each node consists of a buffer connected to a transmitter. Consider two buffers $Q_{1}, Q_{2}$ with transmitters $S_{1}, S_{2}$ which are connected as series in Tandem model. It is assumed that the packet after getting service through first transmitter may join the second buffer which is in series connected to $S_{1}$ or may be returned back to $S_{1}$. The arrival of packets follows 
homogeneous Poisson processes with a mean composite arrival rate $\lambda$. It is further assumed that the packets are transmitted through the transmitter; the mean service rate in the transmitter is linearly dependent on the content of the buffer connected to it. The buffer discipline is First-In First-Out (FIFO). After getting transmitted from the first transmitter the packets are forwarded to the second buffer for transmission with probability (1- $\theta$ ) or returned back to the first buffer with probability $\theta$. The service completion in both the transmitters follows Poisson processes with the parameters $\mu_{1}$ and $\mu_{2}$ for the first and second transmitters. The transmission rate of each packet is adjusted just before transmission depending on the content of the buffer connected to the transmitter. A schematic diagram representing the network model with two transmitters and feedback for first transmitter is shown in figure 1.

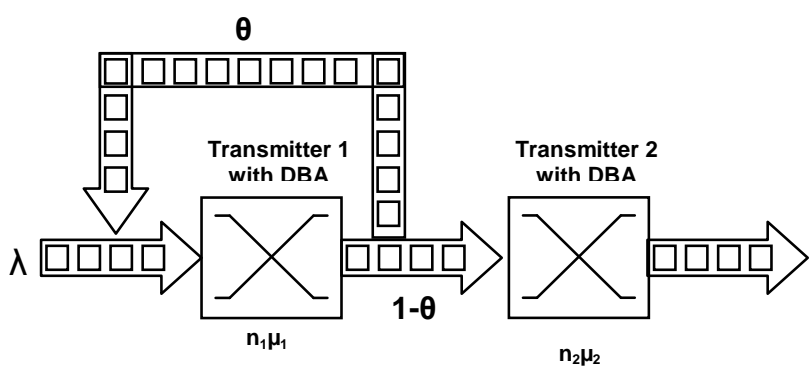

Fig 1: Communication network model with feedback for first node

Let $\mathrm{n}_{1}$ and $\mathrm{n}_{2}$ are the number of packets in first and second buffers and let $P_{n 1, n 2}$ be the probability that there are $n_{1}$ packets in the first buffer and $n_{2}$ packets in the second buffer at time $t$. The difference-differential equations governing the above model are as follows:

$$
\begin{aligned}
\frac{\partial P_{n_{1} n_{2}}(t)}{\partial t}= & -\left(\lambda+n_{1} \mu_{1}(1-\theta)+n_{2} \mu_{2}\right) P_{n_{1}, n_{2}}(t)+\lambda P_{n_{1}-1, n_{2}}(t)+ \\
& \left(n_{1}+1\right) \mu_{1}(1-\theta) P_{n_{1}+1, n_{2}-1}(t)+\left(n_{2}+1\right) \mu_{2} P_{n_{1}, n_{2}+1}(t) \\
\frac{\partial P_{n_{1}, 0}(t)}{\partial t}= & -\left(\lambda+n_{1} \mu_{1}(1-\theta)\right) P_{n_{1}, 0}(t)+\lambda P_{n_{1}-1,0}(t)+\mu_{2} P_{n_{1}, 1}(t) \\
\frac{\partial P_{0, n_{2}}(t)}{\partial t}= & -\left(\lambda+n_{2} \mu_{2}\right) P_{0, n_{2}}(t)+\mu_{1}(1-\theta) P_{n_{1}, n_{2}-1}(t)+ \\
& \left(n_{2}+1\right) \mu_{2} P_{0, n_{2}+1}(t) \\
\frac{\partial P_{0,0}(t)}{\partial t}= & -\lambda P_{0,0}(t)+\mu_{2} P_{0,1}(t)
\end{aligned}
$$

Let $\mathrm{P}\left(\mathrm{S}_{1}, \mathrm{~S}_{2} ; \mathrm{t}\right)$ be the joint probability generating function of $\mathrm{P}_{\mathrm{n} 1, \mathrm{n} 2}(\mathrm{t})$. Then multiply the equation 2.1 with $S_{1}^{n_{1}} S_{2}^{n_{2}}$ and sum over all $\mathrm{n}_{1}, \mathrm{n}_{2}$ to get the following equations.

$$
\begin{aligned}
& \frac{d P\left(s_{1}, s_{2}: t\right)}{d t}=-\lambda P\left(s_{1}, s_{2}: t\right)+\lambda \sum_{n_{1}=0}^{\infty} \sum_{n_{2}=0}^{\infty} P_{n_{1}-1, n_{2}}(t) s_{1}^{n_{1}} s_{2}^{n_{2}}- \\
& \sum_{n_{1}=1}^{\infty} \sum_{n_{2}=0}^{\infty} n_{1} \mu_{1}(1-\theta) P_{n_{1}, n_{2}}(t) s_{1}^{n_{1}} s_{2}^{n_{2}}+ \\
& \sum_{n_{1}=0}^{\infty} \sum_{n_{2}=1}^{\infty}\left(n_{1}+1\right) \mu_{1}(1-\theta) P_{n_{1}+1, n_{2}-1}(t) s_{1}^{n_{1}} s_{2}^{n_{2}}-\sum_{n_{1}=0}^{\infty} \sum_{n_{2}=1}^{\infty} n_{2} \mu_{2} P_{n_{1}, n_{2}}(t) s_{1}^{n_{1}} s_{2}^{n_{2}}+ \\
& \sum_{n_{1}=0}^{\infty} \sum_{n_{2}=1}^{\infty}\left(n_{2}+1\right) \mu_{2} P_{n_{1}, n_{2}+1}(t) s_{1}^{n_{1}} s_{2}^{n_{2}}+\sum_{n_{1}=0}^{\infty} \mu_{2} P_{n_{1}, 1}(t) s_{1}^{n_{1}}
\end{aligned}
$$

After simplifying the resultant equation is,

$$
\begin{aligned}
& \frac{d P\left(s_{1}, s_{2}: t\right)}{d t}=\mu_{1}(1-\theta)\left(s_{2}-s_{1}\right) \frac{\partial p}{\partial s_{1}}+ \\
& \mu_{2}\left(1-s_{2}\right) \frac{\partial p}{\partial s_{2}}-\lambda\left(1-s_{1}\right) \frac{\partial P\left(s_{1}, s_{2}: t\right)}{\partial t}
\end{aligned}
$$

Solving equation 2.3 by Lagrangian's method, we get the auxiliary equations as,

$$
\frac{d t}{1}=\frac{-d s_{1}}{\mu_{1}(1-\theta)\left(s_{2}-s_{1}\right)}=\frac{-d s_{2}}{\mu_{2}\left(1-s_{2}\right)}=\frac{d p}{\lambda P\left(s_{1}-1\right)}
$$

Solve first and third terms of equation 2.4 to get

$a=\left(s_{2}-1\right) e^{-\mu_{2} t}$

Solve first and second terms of equation 2.4 to get

$$
b=\left(s_{1}-1\right) e^{-\mu_{1}(1-\theta) t}+\frac{\left(s_{2}-1\right) \mu_{1}(1-\theta) e^{-\mu_{1}(1-\theta) t}}{\left(\mu_{2}-\mu_{1}(1-\theta)\right)}
$$

Solve first and forth terms of equation 2.4 to get

$$
c=P\left(S_{1}, S_{2} ; t\right) \exp \left\{-\left[\frac{\left(s_{1}-1\right) \lambda}{\mu_{1}(1-\theta)}+\frac{\left(s_{2}-1\right) \lambda}{\mu_{2}}\right]\right\}
$$

Where $a, b$ and $c$ are arbitrary constants.

The general solution of equation 2.4 gives the probability generating function of the number of packets in the first and second buffers at time $\mathrm{t}$, as $\mathrm{P}\left(\mathrm{s}_{1}, \mathrm{~s}_{2} ; \mathrm{t}\right)$.

$$
P\left(\boldsymbol{S}_{1}, \boldsymbol{S}_{2} ; t\right)=\exp \left\{\begin{array}{l}
\frac{\left(s_{1}-1\right) \lambda}{\mu_{1}(1-\theta)}\left(1-e^{-\mu_{1}(1-\theta) t}\right)+\frac{\left(s_{2}-1\right) \lambda\left(1-e^{\mu_{2} t}\right)}{\mu_{2}}+ \\
\frac{\left(s_{2}-1\right) \lambda}{\left(\mu_{2}-\mu_{1}(1-\theta)\right)}\left(e^{\mu_{2} t}-e^{-\mu_{1}(1-\theta) t}\right)
\end{array}\right\}
$$

\section{PERFORMANCE MEASURES OF THE NETWORK MODEL}

In this section, we derive and analyze the performance measures of the communication network under transient conditions. Expanding $\mathrm{P}\left(\mathrm{s}_{1}, \mathrm{~s}_{2} ; \mathrm{t}\right)$ of equation of 2.6 and collecting the constant terms, we get the probability that the network is empty as

$$
P_{00}(t)=\exp \left\{\begin{array}{l}
\frac{-1}{\mu_{1}(1-\theta)} \lambda\left(1-e^{-\mu_{1}(1-\theta) t}\right)+\frac{-1}{\mu_{2}}\left(1-e^{-\mu_{2} t}\right) \lambda+ \\
\frac{-1}{\left(\mu_{2}-\mu_{1}(1-\theta)\right)} \lambda\left(e^{-\mu_{2} t}-e^{-\mu_{1}(1-\theta) t}\right)
\end{array}\right\}
$$

By taking $s_{2}=1$ in equation (2.6), we get the probability generating function of the first buffer size as,

$$
P\left(\mathrm{~s}_{1} ; \mathrm{t}\right)=\exp \left\{\frac{\left(s_{1}-1\right) \lambda}{\mu_{1}(1-\theta)}\left(1-e^{-\mu_{1}(1-\theta) t}\right)\right\}
$$

Expanding the $\mathrm{P}\left(\mathrm{s}_{1} ; \mathrm{t}\right)$ and collecting the constant terms, we get the probability that the first buffer is empty as, 


$$
P_{0 .}(t)=\exp \left\{\frac{-1}{\mu_{1}(1-\theta)} \lambda\left(1-e^{-\mu_{1}(1-\theta) t}\right)\right\}
$$

By taking $\mathrm{s}_{1}=1$ in equation (2.6), we get the probability generating function of the second buffer size as,

$$
P\left(\mathrm{~s}_{2} ; \mathrm{t}\right)=\exp \left\{\begin{array}{l}
\frac{\left(s_{2}-1\right) \lambda\left(1-e^{-\mu_{2} t}\right)}{\mu_{2}}+ \\
\frac{\left(s_{2}-1\right) \lambda}{\left(\mu_{2}-\mu_{1}(1-\theta)\right)}\left(e^{-\mu_{2} t}-e^{-\mu_{1}(1-\theta) t}\right)
\end{array}\right\}
$$

Expanding the $\mathrm{P}\left(\mathrm{s}_{2} ; \mathrm{t}\right)$ and collecting the constant terms, we get the probability that the second buffer is empty as,

$$
P_{.0}(t)=\exp \left\{\begin{array}{l}
\frac{-1}{\mu_{2}}\left(1-e^{-\mu_{2} t}\right) \lambda+ \\
\frac{-1}{\left(\mu_{2}-\mu_{1}(1-\theta)\right)}\left(e^{-\mu_{2} t}-e^{-\mu_{1}(1-\theta) t}\right) \lambda
\end{array}\right\}
$$

The mean number of packets in the first buffer is

$$
L_{1}(t)=\left\{\frac{1}{\mu_{1}(1-\theta)} \lambda\left(1-e^{-\mu_{1}(1-\theta) t}\right)\right\}
$$

The utilization of the first transmitter is

$$
\begin{aligned}
& U_{1}(t)=1-P_{0 .}(t) \\
& =1-\exp \left\{\frac{-1}{\mu_{1}(1-\theta)} \lambda\left(1-e^{-\mu_{1}(1-\theta) t}\right)\right\}
\end{aligned}
$$

The mean number of packets in the second buffer is

$$
L_{2}(t)=\left\{\begin{array}{l}
\frac{1}{\mu_{2}}\left(1-e^{-\mu_{2} t}\right) \lambda+ \\
\frac{1}{\left(\mu_{2}-\mu_{1}(1-\theta)\right)}\left(e^{-\mu_{2} t}-e^{-\mu_{1}(1-\theta) t}\right) \lambda
\end{array}\right\}
$$

The utilization of the second transmitter is

$$
\begin{aligned}
& U_{2}(t)=1-P_{.0}(t) \\
& =1-\exp \left\{\begin{array}{l}
\frac{-1}{\mu_{2}}\left(1-e^{-\mu_{2} t}\right) \lambda+ \\
\frac{-1}{\left(\mu_{2}-\mu_{1}(1-\theta)\right)}\left(e^{-\mu_{2} t}-e^{-\mu_{1}(1-\theta) t}\right) \lambda
\end{array}\right\}
\end{aligned}
$$

The variance of the number of packets in the first buffer is

$$
V_{1}(t)=\left\{\frac{1}{\mu_{1}(1-\theta)} \lambda\left(1-e^{\mu_{1}(1-\theta) t}\right)\right\}
$$

The variance of the number of packets in the second buffer is

$V_{2}(t)=\left\{\begin{array}{l}\frac{1}{\mu_{2}}\left(1-e^{-\mu_{2} t}\right) \lambda+ \\ \frac{1}{\left(\mu_{2}-\mu_{1}(1-\theta)\right)}\left(e^{-\mu_{2} t}-e^{-\mu_{1}(1-\theta) t}\right) \lambda\end{array}\right\}$

The throughput of the first transmitter is

$$
\boldsymbol{\mu}_{1}\left(1-P_{0}(t)\right)
$$

The mean delay in the first buffer is

$$
W_{1}(t)=\frac{L_{1}(t)}{\mu_{1}\left(1-P_{0 .}(t)\right)}
$$

The throughput of the second transmitter is
$\mu_{2}\left(1-P_{.0}(t)\right)$

The mean delay in the second buffer is

$W_{2}(t)=\frac{L_{2}(t)}{\mu_{2}\left(1-P_{.0}(t)\right)}$

The mean number of packets in the entire network at time $t$ is

$\boldsymbol{L}(\boldsymbol{t})=\boldsymbol{L}_{1}(t)+\boldsymbol{L}_{2}(t)$

The variability of the number of packets in the network is

$V(t)=V_{1}(t)+V_{2}(t)$

\section{PERFORMANCE EVALUATION OF THE NETWORK MODEL}

In this section, we discussed the performance of the proposed network model with numerical illustration. Different values of the parameters are taken for bandwidth allocation and arrival of packets. The packet arrival $(\lambda)$ varies from $2 \times 10^{4}$ packets/sec to $7 \times 10^{4}$ packets $/ \mathrm{sec}$, probability parameter $(\theta)$ varies from 0.1 to 0.9 , the transmission rate for first transmitter $\left(\mu_{1}\right)$ varies from $5 \times 10^{4}$ packets/sec to $9 \times 10^{4}$ packets/sec and transmission rate for second transmitter $\left(\mu_{2}\right)$ varies from $15 \times 10^{4}$ packets/sec to $19 \times 10^{4}$ packets/sec. Dynamic Bandwidth Allocation strategy is considered for both the two transmitters. So, the transmission rate of each packet depends on the number of packets in the buffer connected to corresponding transmitter.

The equations 3.7, 3.9, 3.12 and 3.14 are used for computing the utilization of the transmitters and throughput of the transmitters for different values of parameters $t, \lambda, \theta, \mu_{1}, \mu_{2}$ and the results are presented in the Table 1 . The graphs showing the relationship between utilization of the transmitters and throughput of the transmitters are shown in the Figure 2.

It is observed from the Table 1 that, when the time $(\mathrm{t})$ and $\lambda$ increases, the utilization of the transmitters is increasing for the fixed value of other parameters $\theta, \mu_{1}, \mu_{2}$. As the parameter $\theta$ increases from 0.1 to 0.9 , the utilization of first transmitter increases and utilization of the second transmitter decreases, this is due to the number of packets arriving at the second transmitter are decreasing as some of the packets are going back to the first transmitter in feedback. As the transmission rate of the first transmitter $\left(\mu_{1}\right)$ increases from 5 to 9 , the utilization of the first transmitter decreases and the utilization of the second transmitter increases by keeping the other parameters as constant. As the transmission rate of the second transmitter $\left(\mu_{2}\right)$ increases from 15 to 19 , the utilization of the first transmitter is constant and the utilization of the second transmitter decreases by keeping the other parameters as constant.

It is also observed that, when the time $(t)$ increases, the throughput of first and second transmitters is increasing for the fixed values of other parameters. When the parameter $\lambda$ increases from $3 \times 10^{4}$ packets $/ \mathrm{sec}$ to $7 \times 10^{4}$ packets $/ \mathrm{sec}$, the throughput of both transmitters is increasing. As the $\theta$ value increases from 0.1 to 0.9 , the throughput of the first transmitter increases and the throughput of the second transmitter is decreasing. As the transmission rate of the first transmitter $\left(\mu_{1}\right)$ increases from $5 \times 10^{4}$ packets $/ \mathrm{sec}$ to $9 \times 10^{4}$ packets $/ \mathrm{sec}$, the throughput of the first and second transmitters is increasing. The transmission rate of the second transmitter $\left(\mu_{2}\right)$ increases from $15 \times 10^{4}$ packets/sec to $19 \times 10^{4}$ packets/sec and the throughput of the first transmitter is constant and the throughput of the second transmitter is increasing. 
Table 1: Values of Utilization and Throughput of the Network model with DBA and Homogeneous Poisson arrivals

\begin{tabular}{|c|c|c|c|c|c|c|c|c|}
\hline $\mathbf{t}$ & $\boldsymbol{\lambda}$ & $\boldsymbol{\theta}$ & $\boldsymbol{\mu}_{\mathbf{1}}$ & $\boldsymbol{\mu}_{\mathbf{2}}$ & $\mathbf{U}_{\mathbf{1}}(\mathbf{t})$ & $\mathbf{U}_{\mathbf{2}}(\mathbf{t})$ & $\mathbf{T h}_{\mathbf{1}}(\mathbf{t})$ & $\mathbf{T h}_{\mathbf{2}}(\mathbf{t})$ \\
\hline $\mathbf{0 . 1}$ & 2 & 0.1 & 5 & 15 & 0.14875 & 0.02433 & 0.74377 & 0.36495 \\
$\mathbf{0 . 3}$ & 2 & 0.1 & 5 & 15 & 0.28052 & 0.08111 & 1.40260 & 1.21665 \\
$\mathbf{0 . 5}$ & 2 & 0.1 & 5 & 15 & 0.32807 & 0.10711 & 1.64035 & 1.60661 \\
$\mathbf{0 . 7}$ & 2 & 0.1 & 5 & 15 & 0.34649 & 0.11766 & 1.73246 & 1.76483 \\
$\mathbf{0 . 9}$ & 2 & 0.1 & 5 & 15 & 0.35384 & 0.12192 & 1.76918 & 1.82876 \\
\hline 0.5 & $\mathbf{3}$ & 0.1 & 5 & 15 & 0.44921 & 0.15628 & 2.24605 & 2.34418 \\
0.5 & $\mathbf{4}$ & 0.1 & 5 & 15 & 0.54851 & 0.20274 & 2.74255 & 3.04114 \\
0.5 & $\mathbf{5}$ & 0.1 & 5 & 15 & 0.62991 & 0.24665 & 3.14954 & 3.69972 \\
0.5 & $\mathbf{6}$ & 0.1 & 5 & 15 & 0.69663 & 0.28814 & 3.48315 & 4.32202 \\
0.5 & $\mathbf{7}$ & 0.1 & 5 & 15 & 0.75132 & 0.32734 & 3.75662 & 4.91006 \\
\hline 0.5 & 2 & $\mathbf{0 . 1}$ & 5 & 15 & 0.32807 & 0.10711 & 1.64035 & 1.60661 \\
0.5 & 2 & $\mathbf{0 . 3}$ & 5 & 15 & 0.37633 & 0.09799 & 1.88164 & 1.46991 \\
0.5 & 2 & $\mathbf{0 . 5}$ & 5 & 15 & 0.43493 & 0.08379 & 2.17462 & 1.25682 \\
0.5 & 2 & $\mathbf{0 . 7}$ & 5 & 15 & 0.50516 & 0.06140 & 2.52578 & 0.92094 \\
0.5 & 2 & $\mathbf{0 . 9}$ & 5 & 15 & 0.58720 & 0.02558 & 2.93601 & 0.38373 \\
\hline 0.5 & 2 & 0.1 & $\mathbf{5}$ & 15 & 0.32807 & 0.10711 & 1.64035 & 1.60661 \\
0.5 & 2 & 0.1 & $\mathbf{6}$ & 15 & 0.29212 & 0.11252 & 1.75272 & 1.68786 \\
0.5 & 2 & 0.1 & $\mathbf{7}$ & 15 & 0.26203 & 0.11621 & 1.83423 & 1.74315 \\
0.5 & 2 & 0.1 & $\mathbf{8}$ & 15 & 0.23676 & 0.11873 & 1.89411 & 1.78100 \\
0.5 & 2 & 0.1 & $\mathbf{9}$ & 15 & 0.21542 & 0.12047 & 1.93882 & 1.80708 \\
\hline 0.5 & 2 & 0.1 & 5 & $\mathbf{1 5}$ & 0.32807 & 0.10711 & 1.64035 & 1.60661 \\
0.5 & 2 & 0.1 & 5 & $\mathbf{1 6}$ & 0.32807 & 0.10119 & 1.64035 & 1.61908 \\
0.5 & 2 & 0.1 & 5 & $\mathbf{1 7}$ & 0.32807 & 0.09588 & 1.64035 & 1.62994 \\
0.5 & 2 & 0.1 & 5 & $\mathbf{1 8}$ & 0.32807 & 0.09108 & 1.64035 & 1.63949 \\
0.5 & 2 & 0.1 & 5 & $\mathbf{1 9}$ & 0.32807 & 0.08673 & 1.64035 & 1.64794 \\
\hline & & & & & & & \\
\hline
\end{tabular}
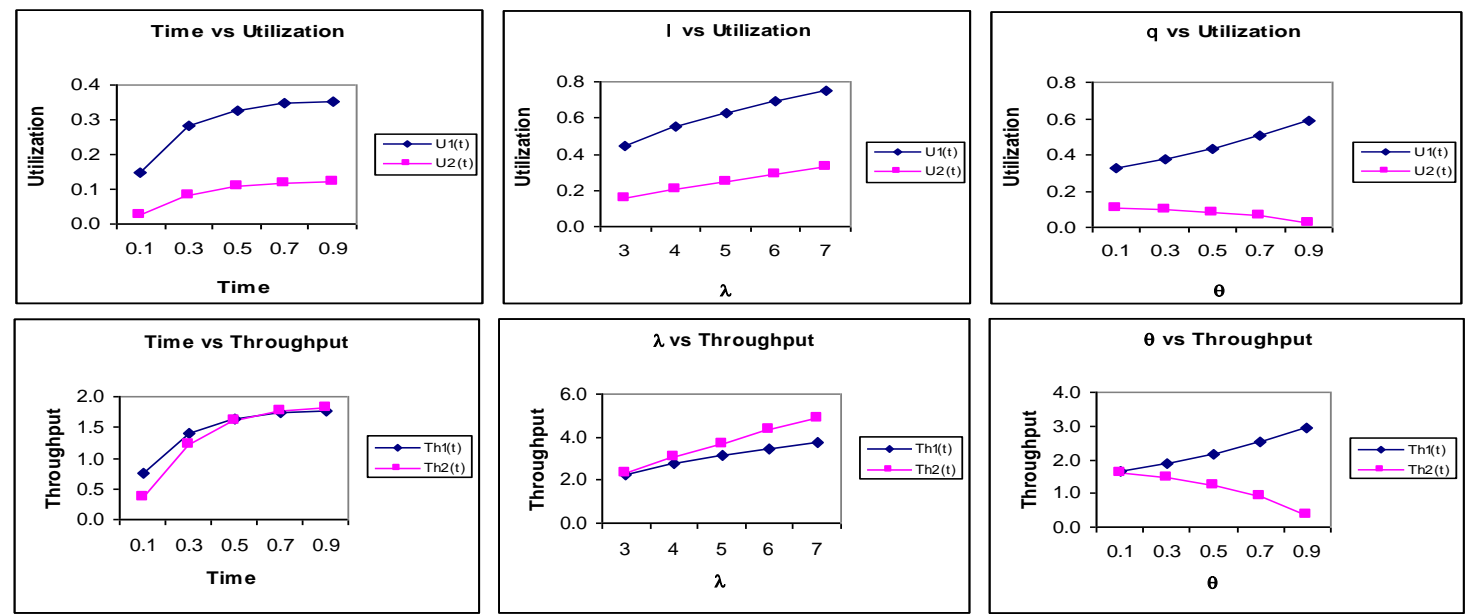

Fig 2: The relationship between Utilization and Throughput and other parameters

Using equations $3.6,3.8,3.16$ and $3.13,3.15$ the mean no. of packets in the buffers and in the network, mean delay in transmission of the two transmitters are calculated for different values of $t, \lambda, \theta, \mu_{1}, \mu_{2}$ and the results are presented in the Table 2. The graphs presenting the relationship between parameters and performance measure are shown in the Figure 3.

It is observed from the Table 2 that as the time $(\mathrm{t})$ varies from 0.1 to 0.9 seconds, the mean number of packets in the two buffers and in the network are increasing when other parameters are kept constant. When the $\lambda$ varies from $3 \times 10^{4}$ packets/second to $7 \times 10^{4}$ packets/second the mean number of packets in the first, second buffers and in the network are increasing. When the parameter $\theta$ varies from 0.1 to 0.9 , the mean number packets in the first buffer increases and decreases in the second buffer due to feedback for the first buffer.

When the transmission rate of the first transmitter $\left(\mu_{1}\right)$ varies from $5 \times 10^{4}$ packets/second to $9 \times 10^{4}$ packets/second, the mean number of packets in the first buffer decreases, in the second buffer increases and in the network decreases. When the transmission rate of the second transmitter $\left(\mu_{2}\right)$ varies from 
$15 \times 10^{4}$ packets/second to $19 \times 10^{4}$ packets/second, the mean number of packets in the first buffer remains constant and decreases in the second buffer and in the network.

It is also observed that with time $(\mathrm{t})$ and $\lambda$, the mean delay in the two buffers are increasing for fixed values of other parameters. When the parameter $\theta$ varies the mean delay in the first buffer increases and decreases in the second buffer due to feedback for the first buffer. As the transmission rate of the first transmitter $\left(\mu_{1}\right)$ varies, the mean delay of the first buffer decreases, and the second buffer increases. When the transmission rate of the second transmitter $\left(\mu_{2}\right)$ varies, the mean delay of the first buffer remains constant and decreases for the second buffer.

According to the above analysis, it is observed that the dynamic bandwidth allocation strategy has a significant influence on all performance measures of the network. We also observed that the performance measures are highly sensitive towards smaller values of time. Hence, it is optimal to consider dynamic bandwidth allocation and evaluate the performance under transient conditions. It is also to be observed that the congestion in buffers and delays in transmission can be reduced to a minimum level by adopting dynamic bandwidth allocation.

Table 2: Values of mean number of packets and mean delay of the network model with DBA and Homogeneous arrivals

\begin{tabular}{|c|c|c|c|c|c|c|c|c|c|}
\hline $\mathbf{t}$ & $\boldsymbol{\lambda}$ & $\boldsymbol{\theta}$ & $\boldsymbol{\mu}_{\mathbf{1}}$ & $\boldsymbol{\mu}_{\mathbf{2}}$ & $\mathbf{L}_{\mathbf{1}}(\mathbf{t})$ & $\mathbf{L}_{\mathbf{2}}(\mathbf{t})$ & $\mathbf{L}(\mathbf{t})$ & $\mathbf{W}_{\mathbf{1}}(\mathbf{t})$ & $\mathbf{W}_{\mathbf{2}}(\mathbf{t})$ \\
\hline $\mathbf{0 . 1}$ & 2 & 0.1 & 5 & 15 & 0.161054 & 0.024631 & 0.185685 & 0.216538 & 0.067491 \\
$\mathbf{0 . 3}$ & 2 & 0.1 & 5 & 15 & 0.329227 & 0.084589 & 0.413816 & 0.234726 & 0.069526 \\
$\mathbf{0 . 5}$ & 2 & 0.1 & 5 & 15 & 0.397600 & 0.113289 & 0.510889 & 0.242388 & 0.070514 \\
$\mathbf{0 . 7}$ & 2 & 0.1 & 5 & 15 & 0.425399 & 0.125173 & 0.550572 & 0.245547 & 0.070926 \\
$\mathbf{0 . 9}$ & 2 & 0.1 & 5 & 15 & 0.436701 & 0.130015 & 0.566716 & 0.246839 & 0.071094 \\
\hline 0.5 & $\mathbf{3}$ & 0.1 & 5 & 15 & 0.596401 & 0.169933 & 0.766334 & 0.265533 & 0.072491 \\
0.5 & $\mathbf{4}$ & 0.1 & 5 & 15 & 0.795201 & 0.226578 & 1.021778 & 0.289950 & 0.074504 \\
0.5 & $\mathbf{5}$ & 0.1 & 5 & 15 & 0.994001 & 0.283222 & 1.277223 & 0.315602 & 0.076552 \\
0.5 & $\mathbf{6}$ & 0.1 & 5 & 15 & 1.192801 & 0.339867 & 1.532668 & 0.342449 & 0.078636 \\
0.5 & $\mathbf{7}$ & 0.1 & 5 & 15 & 1.391601 & 0.396511 & 1.788112 & 0.370440 & 0.080755 \\
\hline 0.5 & 2 & $\mathbf{0 . 1}$ & 5 & 15 & 0.397600 & 0.113289 & 0.510889 & 0.242388 & 0.070514 \\
0.5 & 2 & $\mathbf{0 . 3}$ & 5 & 15 & 0.472129 & 0.103134 & 0.575263 & 0.250914 & 0.070164 \\
0.5 & 2 & $\mathbf{0 . 5}$ & 5 & 15 & 0.570796 & 0.087507 & 0.658303 & 0.262480 & 0.069626 \\
0.5 & 2 & $\mathbf{0 . 7}$ & 5 & 15 & 0.703511 & 0.063361 & 0.766873 & 0.278533 & 0.068801 \\
0.5 & 2 & $\mathbf{0 . 9}$ & 5 & 15 & 0.884797 & 0.025915 & 0.910712 & 0.301360 & 0.067534 \\
\hline 0.5 & 2 & 0.1 & $\mathbf{5}$ & 15 & 0.397600 & 0.113289 & 0.510889 & 0.242388 & 0.070514 \\
0.5 & 2 & 0.1 & $\mathbf{6}$ & 15 & 0.345479 & 0.119374 & 0.464853 & 0.197111 & 0.070725 \\
0.5 & 2 & 0.1 & $\mathbf{7}$ & 15 & 0.303856 & 0.123536 & 0.427392 & 0.165659 & 0.070869 \\
0.5 & 2 & 0.1 & $\mathbf{8}$ & 15 & 0.270188 & 0.126395 & 0.396583 & 0.142646 & 0.070969 \\
0.5 & 2 & 0.1 & $\mathbf{9}$ & 15 & 0.242612 & 0.128370 & 0.370982 & 0.125134 & 0.071037 \\
\hline 0.5 & 2 & 0.1 & 5 & $\mathbf{1 5}$ & 0.397600 & 0.113289 & 0.510889 & 0.242388 & 0.070514 \\
0.5 & 2 & 0.1 & 5 & $\mathbf{1 6}$ & 0.397600 & 0.106686 & 0.504286 & 0.242388 & 0.065893 \\
0.5 & 2 & 0.1 & 5 & $\mathbf{1 7}$ & 0.397600 & 0.100792 & 0.498392 & 0.242388 & 0.061838 \\
0.5 & 2 & 0.1 & 5 & $\mathbf{1 8}$ & 0.397600 & 0.095501 & 0.493101 & 0.242388 & 0.058251 \\
0.5 & 2 & 0.1 & 5 & $\mathbf{1 9}$ & 0.397600 & 0.090728 & 0.488328 & 0.242388 & 0.055055 \\
\hline
\end{tabular}
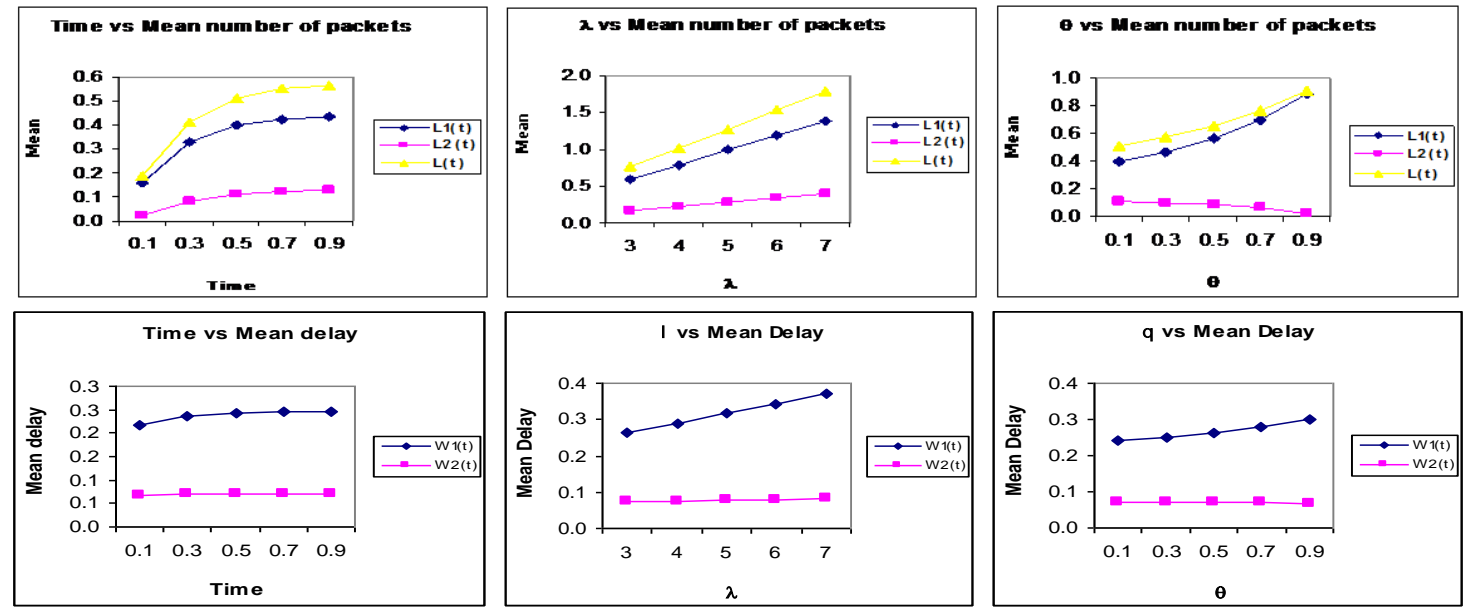

Fig 3: The relationship between mean no. of packets, mean delay and various parameters 


\section{SENSITIVITY ANALYSIS}

This section consists of the sensitivity analysis of the model with respect to the changes in the parameters $t, \lambda$, and $\theta$ on the mean number of packets, utilization of the transmitters, mean delay and throughput of the first and second transmitters. The values considered for the sensitivity analysis are, $\mathrm{t}=0.5 \mathrm{sec}, \lambda=$ $2 \times 10^{4}$ packets $/ \mathrm{sec}, \mu_{1}=5 \times 10^{4}$ packets $/$ second, to $\mu_{2}=15 \times 10^{4}$ packets/second and $\theta=0.1$. The mean number of packets, utilization of the transmitters, mean delay and throughput of the first and second transmitters are computed with variation of $(-15) \%,(-10) \%,(-5) \%, 0 \%,+5 \%,+10 \%,+15 \%$ on the model and are presented in the Table 3 . The performance measures are highly affected by the changes in the values of time ( $t$ ), arrival and probability $(\theta)$.

As the time $(\mathrm{t})$ increases from $-15 \%$ to $+15 \%$ the average number of packets in the two buffers increase along with the average delay in buffers, the utilization and throughput of the transmitters. As the arrival parameter $(\lambda)$ increases from $-15 \%$ to $+15 \%$ the average number of packets in the two buffers increase along with the average delay in buffers, the utilization and throughput of the transmitters. As the probability parameter $(\theta)$ increases from $-15 \%$ to $+15 \%$ the average number of packets in the first buffer increase along with the average delay in buffers, the utilization and throughput of the transmitter. But average number of packets in the second buffer decrease along with the average delay in buffers, the utilization and throughput of the transmitter due to feedback for the first transmitter.

From the above analysis it is observed that the dynamic bandwidth allocation strategy has an important influence on all performance measures of the network. It is also observed that these performance measures are also sensitive towards the probability parameter $(\theta)$ that causes feedback of packets to the first transmitter.

\section{CONCLUSION}

This paper deals with a two node tandem network model with dynamic bandwidth allocation and feedback for the first transmitter. The dynamic bandwidth allocation is adapted by immediate adjustment of packet service time by utilizing idle bandwidth in the transmitter. The transient analysis of the model is capable of capturing the changes in the performance measures of the network like average content of the buffers, mean delays, throughput of the transmitters, idleness of the transmitters etc, explicitly. The numerical study reveals that the proposed communication network model is capable of evaluating and predicting the performance of network more close to the reality. It is possible to extent this network model to non homogeneous Poisson arrivals.

Table 3: Sensitivity analysis of the network model

\begin{tabular}{|c|c|c|c|c|c|c|c|c|}
\hline \multirow{2}{*}{ Parameter } & \multirow{2}{*}{$\begin{array}{c}\text { Performance } \\
\text { Measure }\end{array}$} & \multicolumn{7}{|c|}{$\%$ change in Parameter } \\
\hline & & -15 & -10 & -5 & $\mathbf{0}$ & 5 & 10 & 15 \\
\hline \multirow{8}{*}{$\mathrm{t}=0.5$} & $\mathrm{~L}_{1}(\mathrm{t})$ & 0.378795 & 0.385781 & 0.392023 & 0.397600 & 0.402585 & 0.407039 & 0.411019 \\
\hline & $\mathrm{L}_{2}(\mathrm{t})$ & 0.105295 & 0.108259 & 0.110913 & 0.113289 & 0.115415 & 0.117317 & 0.119018 \\
\hline & $\mathrm{U}_{1}(\mathrm{t})$ & 0.315314 & 0.320080 & 0.324311 & 0.328069 & 0.331410 & 0.334382 & 0.337025 \\
\hline & $\mathrm{U}_{2}(\mathrm{t})$ & 0.099941 & 0.102604 & 0.104983 & 0.107107 & 0.109004 & 0.110697 & 0.112208 \\
\hline & $\mathrm{Th}_{1}(\mathrm{t})$ & 1.576571 & 1.600401 & 1.621555 & 1.640347 & 1.657051 & 1.671908 & 1.685127 \\
\hline & $\mathrm{Th}_{2}(\mathrm{t})$ & 1.499120 & 1.539067 & 1.574748 & 1.606610 & 1.635058 & 1.660454 & 1.683127 \\
\hline & $\mathrm{W}_{1}(\mathrm{t})$ & 0.240265 & 0.241052 & 0.241757 & 0.242388 & 0.242952 & 0.243458 & 0.243910 \\
\hline & $\mathrm{W}_{2}(\mathrm{t})$ & 0.070238 & 0.070340 & 0.070432 & 0.070514 & 0.070588 & 0.070654 & 0.070713 \\
\hline \multirow{8}{*}{$\lambda=2$} & $\mathrm{~L}_{1}(\mathrm{t})$ & 0.337960 & 0.357840 & 0.377720 & 0.397600 & 0.417480 & 0.437360 & 0.457240 \\
\hline & $\mathrm{L}_{2}(\mathrm{t})$ & 0.096296 & 0.101960 & 0.107624 & 0.113289 & 0.118953 & 0.124618 & 0.130282 \\
\hline & $\mathrm{U}_{1}(\mathrm{t})$ & 0.286776 & 0.300815 & 0.314578 & 0.328069 & 0.341296 & 0.354261 & 0.366972 \\
\hline & $\mathrm{U}_{2}(\mathrm{t})$ & 0.091804 & 0.096934 & 0.102035 & 0.107107 & 0.112151 & 0.117166 & 0.122152 \\
\hline & $\mathrm{Th}_{1}(\mathrm{t})$ & 1.433882 & 1.504076 & 1.572889 & 1.640347 & 1.706478 & 1.771307 & 1.834859 \\
\hline & $\mathrm{Th}_{2}(\mathrm{t})$ & 1.377067 & 1.454015 & 1.530529 & 1.606610 & 1.682262 & 1.757486 & 1.832285 \\
\hline & $\mathrm{W}_{1}(\mathrm{t})$ & 0.235696 & 0.237914 & 0.240144 & 0.242388 & 0.244644 & 0.246914 & 0.249196 \\
\hline & $\mathrm{W}_{2}(\mathrm{t})$ & 0.069928 & 0.070123 & 0.070318 & 0.070514 & 0.070710 & 0.070907 & 0.071104 \\
\hline \multirow{8}{*}{$\theta=0.1$} & $\mathrm{~L}_{1}(\mathrm{t})$ & 0.392778 & 0.394375 & 0.395982 & 0.397600 & 0.399229 & 0.400869 & 0.402519 \\
\hline & $\mathrm{L}_{2}(\mathrm{t})$ & 0.113889 & 0.113691 & 0.113491 & 0.113289 & 0.113084 & 0.112878 & 0.112669 \\
\hline & $\mathrm{U}_{1}(\mathrm{t})$ & 0.324821 & 0.325899 & 0.326981 & 0.328069 & 0.329163 & 0.330262 & 0.331366 \\
\hline & $\mathrm{U}_{2}(\mathrm{t})$ & 0.107643 & 0.107467 & 0.107288 & 0.107107 & 0.106925 & 0.106740 & 0.106554 \\
\hline & $\mathrm{Th}_{1}(\mathrm{t})$ & 1.624107 & 1.629494 & 1.634907 & 1.640347 & 1.645815 & 1.651310 & 1.656832 \\
\hline & $\mathrm{Th}_{2}(\mathrm{t})$ & 1.614651 & 1.612000 & 1.609319 & 1.606610 & 1.603871 & 1.601102 & 1.598303 \\
\hline & $\mathrm{W}_{1}(\mathrm{t})$ & 0.241842 & 0.242023 & 0.242205 & 0.242388 & 0.242572 & 0.242758 & 0.242945 \\
\hline & $\mathrm{W}_{2}(\mathrm{t})$ & 0.070535 & 0.070528 & 0.070521 & 0.070514 & 0.070507 & 0.070500 & 0.070493 \\
\hline
\end{tabular}




\section{REFERENCES}

[1] D. Ferrari. G. Serazzi. and A. Zeigner, Measurement and Turning of Computer Systems.

[2] E. Gelenbe and I. Mitrani, Analysis and Synthesis of Computer System Models. London: Academic, 1980.

[3] C. H. Sauer and K. M. Chandy, Computer Systems Performance Modeling. Englewood Cliffs, NJ PrenticeHall, 1981

[4] Gaujal, B. and Hyon, E., Optimal routing policies in deterministic queues in tandem, Proceedings of Sixth International Workshop on Discrete Event Systems (WODES'02),pp 251-257, 2002.

[5] Parthasarathy, P.R. and Selvraju, N., Transient analysis of a Queue where potential customers are discouraged by Queue length. Mathematical Problems in Engineering, Vol.7, pp.433-454, 2001

[6] Kleinrock L., Queuing system, vol.II, Computer Applications, wiley publications, Newyork, 1976.

[7] Yukuo Hayashida, Throughput analysis of tandem type goback NARQ scheme for satellite communications, IEEE Transactions on communication, Vol.41, pp 1517-1524, 1993.

[8] Sriram K., Methodologies for bandwidth allocation, transmission scheduling and congestion control avoidance in broadband ATM Networks, computer Network, ISDN system, j.26, pp. 43-59, 1993.

[9] P.Suresh Varma and K.Srinivasa Rao, A Communication network with load dependent transmission, International Journal of Mathematical Sciences, Vol.6, No.2, pp 199210, 2007.

[10] Nageswara Rao K., K. Srinivas Rao, P Srinivasa Rao, A tandem communication Network with Dynamic Bandwidth Allocation and modified phase type transmission having Bulk arrivals, International Journal of Computer science Issues, Vol.7, No.3, pp 18-26, 2010.
[11] Padmavathi G., K. Srinivas Rao, KVVS Reddy, Performance Evaluation of parallel and series communication Network with Dynamic Bandwidth Allocation, CIIT International Journal of Networking and communication Engineering Vol. 1, No. 7, pp 410-421, 2009.

[12] Nageswara Rao K., K. Srinivas Rao, P Srinivasa Rao, Transient Analysis of a Tandem Communication Network with Dynamic Bandwidth Allocation having Two Stage Direct Bulk Arrivals, International Journal of Computer Applications (0975 - 8887) Vol. 13, No.7, January 2011.

CH.V. Raghavendran working as an Associate Professor in P.G. Dept. of Computer Sciences, Ideal College of Arts \& Sciences, Kakinada, AP, India. He is a research scholar in Computer Science Department of Adikavi Nannaya University, Rajahmundry, AP, India. He has presented several papers in various National and International Conferences.

Ganti Naga Satish is working as an Associate Professor in P.G Department of Computer Sciences, Ideal College of Arts \& Sciences, Kakinada, AP, India. He is a research scholar in Computer Science Department of Adikavi Nannaya University, Rajahmundry, AP, India. He has presented and published papers in National and International Conferences.

Dr. M.V.Rama Sundari is working as an Associate Professor Department of IT, Godavari Institute of Engineering and Technology, Rajahmundry, AP, India. She published several research papers in national and international journals.

Dr. P.Suresh Varma is presently working as Professor in the Department of Computer Science, Adikavi Nannaya University, Rajahmundry, India. He published several research papers in national and international journals and presented papers at various conferences, seminars and workshops. He guided a large number of students for their M.Tech, MCA degrees in Computer Science and Engineering and Information Technology. His current research interests are Communication Networks, Data Mining and Cloud Computing. 